\title{
Liver dose reduction by deep inspiration breath hold technique in right-sided breast irradiation
}

\author{
Gunel Haji, Ulviye Nabizade, Kamal Kazimov, Naile Guliyeva, Isa Isayev \\ Department of Radiation Oncology, National Center of Oncology, Baku, Azerbaijan
}

Purpose: Deep inspiration breath hold (DIBH) is a well-established technique that enables efficient cardiac sparing in patients with left-sided breast cancer. The aim of the current study was to determine if DIBH is effective for reducing radiation exposure of of liver and other organs at risk in right breast radiotherapy (RT).

Materials and Methods: Twenty patients with right-sided breast cancer were enrolled in this study. Three-dimensional conformal RT plans were generated for each patient, with two different computed tomography scans of free breathing (FB) and DIBH. Nodes were contoured according to the Radiation Therapy Oncology Group contouring guidelines. Dose-volume histograms for the target volume coverage and organs at risk were evaluated and analyzed.

Results: DIBH plans showed significant reduction in mean liver dose ( $5.59 \pm 2.07$ Gy vs. $2.54 \pm 1.40 \mathrm{~Gy} ; \mathrm{p}=0.0003), \mathrm{V}_{20 \mathrm{~Gy}}(148.38$ \pm 73.05 vs. $64.19 \pm 51.07 \mathrm{~mL} ; \mathrm{p}=0.0003)$ and $\mathrm{V}_{106 \mathrm{y}}(195.34 \pm 93.57$ vs. $89.81 \pm 57.28 \mathrm{~mL} ; \mathrm{p}=0.0003)$ volumes compared with FB plans. Right lung doses were also significantly reduced in DIBH plans. Heart and left lung doses showed small but statistically significant improvement with application of the DIBH technique.

Conclusion: We report that the use of DIBH for right-sided breast cancer significantly reduces the radiation doses to the liver, lungs, and heart.

Keywords: Breast cancer, Deep inspiration breath-hold, Liver, Radiotherapy

\section{Introduction}

Radiotherapy (RT) is a key part of breast cancer management. It results in improvement in loco-regional control and overall survival [1]. As survival improves, side effects of RT may outweigh risks related to the primary disease and become the main determinant of survival $[2,3]$. Therefore, various techniques have been developed to minimise exposure of the surrounding normal tissues and consequently short- and longterm side effects. One of these is the deep inspiration breath hold (DIBH) technique, which has been shown to reduce radiation exposure of the cardiac chambers and coronaries in patients with left-sided breast cancer (LBC) [4-7]. Numerous dosimetric studies clearly support the technique in patients with $L B C$, and it is hoped that this effect will translate into clinical benefits [4-8]. DIBH is widely accepted as an important tool in selected patients to prevent long-term cardiovascular sequelae of RT.

Despite the benefits in patients with LBC, utility of the technique is not well established in patients with right-sided

Received 17 April 2019, Revised 23 June 2019, Accepted 29 July 2019.

Correspondence: Gunel Haji, Department of Radiation Oncology, National Center of Oncology, 79B, H. Zardabi street, Baku AZ1012, Azerbaijan. Tel: +994-12-537-08-11, Fax: +994-55-626-26-52, E-mail: gunelhaci@yahoo.com (http://orcid.org/00000002-1325-1218)

(c) This is an Open Access article distributed under the terms of the Creative Commons Attribution Non-Commercial License (http://creativecommons.org/ licenses/by-nc/4.0/) which permits unrestricted non-commercial use, distribution, and reproduction in any medium, provided the original work is properly cited.

www.e-roj.org 
breast cancer (RBC). The primary aim of our study was to assess the dosimetric benefits of DIBH compared to standard free breathing (FB) approaches in terms of liver doses for RBC patients following mastectomy. Previous studies exclusively focused on patients with $L B C$, and just two recent studies have examined the benefit and applicability of DIBH in patients with $\operatorname{RBC}[9,10]$. Although these studies provide evidence in this field, they are not without shortcomings. Firstly, liver doses were not addressed by Essers et al. [9] in the first study. Another study that provided dosimetric comparison of liver doses between the techniques was conducted on LBC patients, as intact right breast was assumed to be representative of breast-conserving surgery for dosimetry purposes [10]. On the other hand, both studies $[9,10]$ followed the European Society for Radiotherapy and Oncology (ESTRO) breast and nodal contouring guidelines, involving larger lung volumes in the treatment field than standard clinical practice [11]. We preferred to use the Radiation Therapy Oncology Group (RTOG) contouring guidelines, which are more widely used in clinical practice [12]. In addition, we aimed to compare heart and ipsilateral lung doses between the techniques.

\section{Materials and Methods}

The study population consisted of 30 consecutive female patients with stage II-III RBC, who were referred for adjuvant RT following mastectomy and agreed to be treated with this technique between January 2018 and May 2018.

Patients older than 70 years (4 patients) with body mass index (BMI) less than $25 \mathrm{~kg} / \mathrm{m}^{2}$ (2 patients) or who were unable to cooperate in the DIBH training (4 patients) were excluded.

Patients were placed supine with the arms over the head and immobilized on the breast board. Patients were given adequate training to hold their breath. All received two planning computed tomography (CT) scans, one in $\mathrm{FB}$ and one in $\mathrm{DIBH}$, each with a slice thickness of $3.0 \mathrm{~mm}$. The DIBH and FB scans were taken on the same day without changing patient position between scans. We used the Sentinel system (C-RAD, Uppsala, Sweden), a laser-based optical surface scanning system used during $\mathrm{CT}$ acquisition to create a reference surface scan in FB and to track deep inspiration amplitude. Three-dimensional (3D) optical glasses were used to maintain a stable gating level.

CT data were transferred to the Varian Eclipse treatment planning system version 13.0 (Varian Medical System, Palo Alto, CA). Clinical target volume (CTV) and normal tissues were delineated on all CT slices of both CT sets for each patient according to the RTOG breast cancer atlas for radiation therapy planning guidelines [12]. The following normal structures were contoured: patient body, contralateral breast, liver, heart, left lung and right lung. CTV was defined as chest wall and regional lymph nodes including supraclavicular, infraclavicular, axillary and internal mammary lymph nodes. Planning target volume (PTV) was created with an additional 5-mm margin on CTV. PTV was retracted $3 \mathrm{~mm}$ from the skin surface. The prescribed dose to the target was 50 Gy in 25 fractions.

For each CT dataset, a 3D conformal RT treatment plan was designed using opposing tangential fields with field-in-field technique, with a mixture of $6 \mathrm{MV}$ and $15 \mathrm{MV}$ photons. Plans were calculated in Eclipse version 13.0. For consistency, the same dosimetrist planned paired CT data sets (FB and DIBH scans) for each patient.

Dose-volume histograms were extracted for both datasets and analysed comparatively. Primary endpoints were dosimetric measures: mean dose of the liver, and liver volumes receiving $\geq 10$ Gy $\left(\mathrm{V}_{10 \mathrm{~Gy}}\right)$ and $\geq 20 \mathrm{~Gy}\left(\mathrm{~V}_{20 \mathrm{~Gy}}\right)$. Additionally, mean dose of the ipsilateral lung, percentage of right lung volume receiving $\geq 20 \mathrm{~Gy}$ and $\geq 30 \mathrm{~Gy}$, mean dose of heart, volume of heart receiving $\geq 5$ Gy $\left(V_{5 G y}\right)$, and left lung $V_{5 G y}$ volume were also compared.

Our study was determined to be a minimal risk and consistent with a quality improvement project using the Alberta Research Ethics Community Consensus Initiative (ARECCI) screening tool provided by the Health Research Ethics Board of Alberta and did not require further ethics board approval [13].

Continuous variables are presented as a mean and standard deviation. The comparisons between the groups were performed using two-tailed pairwise Wilcoxon signed rank tests. All statistical computations were carried out using Statistical Package for the Social Sciences (SPSS) version 17 (SPSS Inc., Chicago, IL, USA). The $p$-value of $<0.05$ was considered to indicate statistically significant difference.

\section{Results}

After applying exclusion criteria, data of 20 patients were analyzed. The median age of the patients was 53 years (range, 35 to 64 years). Eleven patients (55\%) were in stage II, and the remaining 9 were in stage III. Pathological diagnosis was invasive ductal carcinoma in 13 patients (65\%) and invasive lobular carcinoma in 7 patients. Mean body weight was 77.8 $\mathrm{kg}$, while mean BMI was $30.4 \mathrm{~kg} / \mathrm{m}^{2}$. All patients finished approximately 20 minutes of training to adequately apply the DIBH method. All 20 patients were treated with DIBH. 
Table 1. Comparison of volume and dosimetric data for free breathing and DIBH technique plans

\begin{tabular}{|c|c|c|c|}
\hline & Free breathing & $\mathrm{DIBH}$ & $p$-value \\
\hline PTV V ${ }_{90 \%}(\%)$ & 95 & 95 & 0.66 \\
\hline Liver mean dose (Gy) & $5.59 \pm 2.07$ & $2.54 \pm 1.40$ & 0.0003 \\
\hline Liver $\mathrm{V}_{20 \mathrm{~Gy}}(\mathrm{~mL})$ & $148.38 \pm 73.05$ & $64.19 \pm 51.07$ & 0.0003 \\
\hline Liver $\mathrm{V}_{10 \mathrm{~Gy}}(\mathrm{~mL})$ & $195.34 \pm 93.57$ & $89.81 \pm 57.28$ & 0.0003 \\
\hline Right lung volume (mL) & $1,254.3 \pm 248.2$ & $2,092.5 \pm 407.9$ & 0.007 \\
\hline Right lung mean dose (Gy) & $16.52 \pm 2.42$ & $13.77 \pm 2.71$ & 0.0001 \\
\hline Right lung $\bigvee_{306 y}(\%)$ & $26.84 \pm 3.50$ & $21.50 \pm 3.11$ & 0.009 \\
\hline Right lung $\mathrm{V}_{20 G y}(\%)$ & $31.15 \pm 3.75$ & $26.53 \pm 4.03$ & 0.0002 \\
\hline Left lung $\mathrm{V}_{5 G y}(\mathrm{~mL})$ & $0.52 \pm 0.91$ & $0.22 \pm 0.30$ & 0.00001 \\
\hline Heart mean dose (Gy) & $1.49 \pm 0.72$ & $1.01 \pm 0.31$ & 0.002 \\
\hline Heart $\bigvee_{5 G y}(\%)$ & $2.00 \pm 1.29$ & $1.24 \pm 0.96$ & 0.0003 \\
\hline
\end{tabular}

Values are presented as mean \pm standard deviation.

DIBH, deep inspiration breath-hold; PTV, planned target volume.

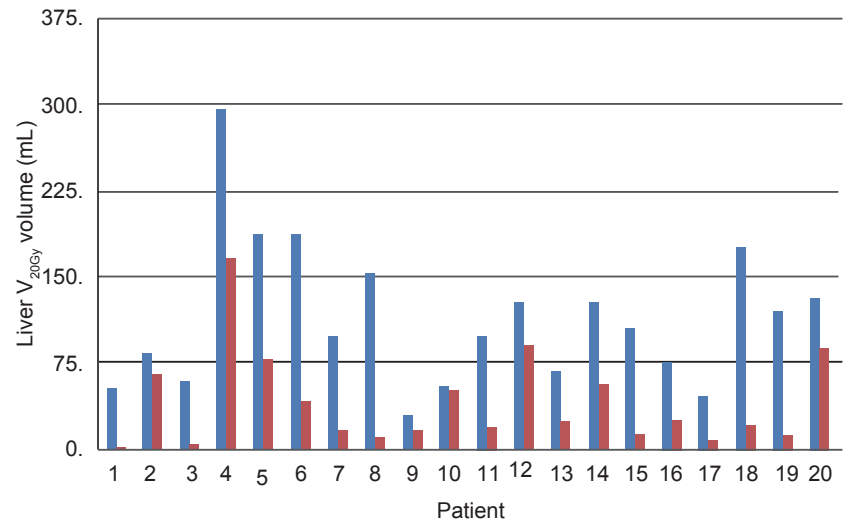

Fig. 1. Liver $\mathrm{V}_{20 \mathrm{~Gy}}$ volumes for $\mathrm{FB}$ (blue bars) and DIBH (red bars) scans for individual patients. FB, free breathing; $\mathrm{DIBH}$, deep inspiration breath-hold.

PTV coverage was similar for DIBH and FB: $V_{90 \%}$ was 95\% for both plans ( $p=0.66)$. Table 1 summarizes the dosimetric comparison of FB and DIBH plans.

The most important outcome parameter in our study was liver exposure, which was significantly lower with DIBH when compared with FB. While liver mean dose with FB was 5.59 $\pm 2.07 \mathrm{~Gy}$, it was markedly decreased with $\mathrm{DIBH}(2.54 \pm 1.40$ Gy; $p=0.0003)$. DIBH scans showed significant reduction in liver $V_{20 G y}$ volume, which was $148.38 \pm 73.05 \mathrm{~mL}$ and $64.19 \pm$ $51.07 \mathrm{~mL}$ for FB and DIBH scans, respectively. Changes in liver $V_{20 G y}$ volume for individual patients are depicted in Fig. 1. Liver $V_{10 G y}$ volume was also reduced with application of the DIBH technique (89.81 \pm 57.28 vs. $195.34 \pm 93.57 \mathrm{~mL} ; \mathrm{p}=0.0003)$. Fig. 2 demonstrates the effects of $\mathrm{DIBH}$ on the surrounding
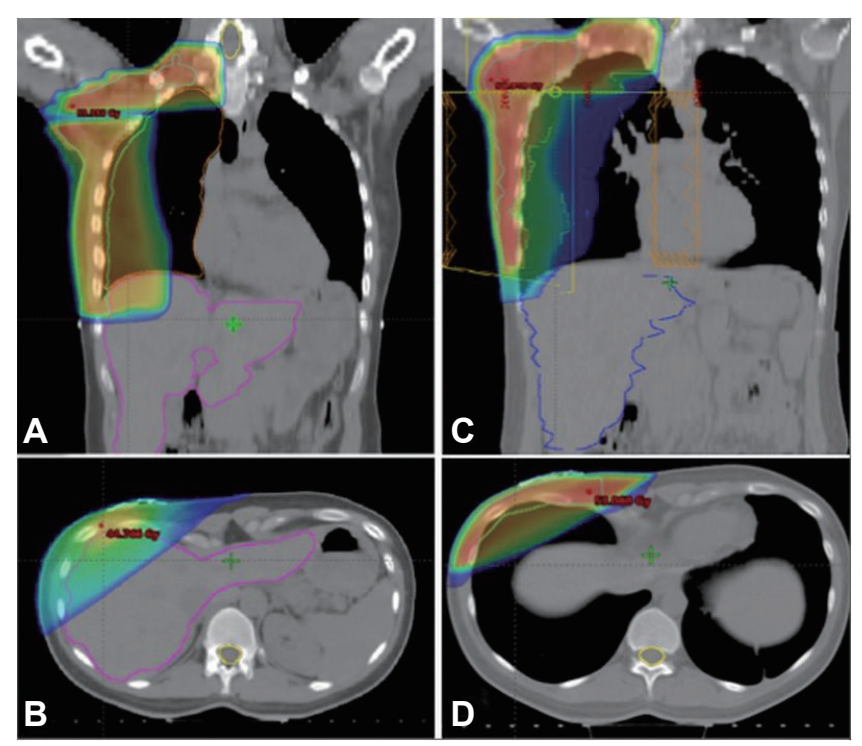

Fig. 2. Computed tomography simulation coronal and axial images of the patient \#3 at $F B$ ( $A$ and $B$, respectively) and $D I B H$ technique ( $C$ and $D$, respectively). $F B$, free breathing; $D I B H$, deep inspiration breath-hold.

organs in one study patient.

The right lung volume was significantly larger with $\mathrm{DIBH}$ than $\mathrm{FB}(2,092.5 \pm 407.9 \mathrm{~mL}$ and $1,254.2 \pm 248.2$ $\mathrm{mL}$, respectively; $p=0.0003)$. The right lung exposure was significantly reduced with application of DIBH in terms of mean dose, $V_{20}$ and $V_{30}$. DIBH also resulted in significant reduction of contralateral lung exposure in terms of $V_{5 G y}$ volume $(0.52 \pm 0.9$ vs. $0.22 \pm 0.30 \mathrm{~mL} ; p=0.00001)$. Although cardiac exposure was already very small in patients with $\mathrm{RBC}$, 
application of DIBH could cause a statistically significant decrease in mean dose and $V_{5}$ volume of the heart.

\section{Discussion and Conclusion}

Since the first publication on DIBH [4], plenty of evidence has supported its effectiveness for reduction of cardiac and other surrounding organ doses in patients with LBC. These studies have changed daily practice in irradiation of LBC patients, and $\mathrm{DIBH}$ technique has become a mainstay in treating selected LBC patients. As the overwhelming majority of these studies consisted of patients with $\angle B C$, with limited work relating to $\operatorname{RBC}[9,10]$, the effectiveness of DIBH in patients with RBC has not been sufficiently investigated.

The present study demonstrated that the DIBH technique is effective in reducing liver dose exposure in patients with RBC. Onlyone other study has investigated DIBH particularly in patients with $\mathrm{RBC}$, but liver doses were not specifically addressed in this work [9]. Another study by Conway et al. [10] investigated liver doses in patients with LBC. For dosimetry purposes, the intact right breast was assumed to be representative of breast-conserving surgery with an excellent cosmetic result [10]. This study showed significant reduction of the liver volume, receiving $25 \mathrm{~Gy}$ and more with application of DIBH technique (7.3 and $49.6 \mathrm{~mL}$, respectively).

Our study included RBC patients following mastectomy, which causes excessive exposure of liver and lung tissues in comparison with patients following breast-conserving surgery as in the aforementioned study [10]. Although liver radiation exposure was already much lower in the FB group $(5.59 \pm$ $2.07 \mathrm{~Gy}$ ) than dose limits (28 Gy), we believe that every effort should be made to minimise normal tissue exposure without compromising PTV coverage.

Hormati et al. [14] found no meaningful relation between breast cancer RT and liver fibrosis, as assessed by elastography, and concluded that breast RT even at doses higher than $40 \mathrm{~Gy}$ had no effect on liver stiffness. However, we must consider that currently available methods may not be sensitive enough or correctly selected to detect radiation-induced liver damage. In cases of liver metastasis to be treated with stereotactic RT or stereotactic body RT in addition to breast irradiation, patients who have already received hepatotoxic chemotherapy regimens, or patients with metachronous or synchronous liver tumours, further liver dose reduction may be beneficial even if these doses are currently considered safe. Because our study was dosimetric, nausea and other manifestations of acute toxicity were not objectives or pre- specified endpoints. However, we observed that nausea seemed to be less frequent compared with conventional RT. We are conducting another study which will examine this issue. Another important result of the current study is ipsilateral lung dose reduction with the DIBH technique. DIBH during radiotherapy significantly reduced average mean dose and mean percentage of volume receiving $\geq 20 \mathrm{~Gy}\left(\mathrm{~V}_{20 \mathrm{~Gy}}\right)$ and $\geq 30 \mathrm{~Gy}\left(\mathrm{~V}_{300 \mathrm{y}}\right)$ compared with $\mathrm{FB}$, in line with previous studies $[9,10]$. Reducing lung exposure in patients with breast cancer is important to prevent radiation pneumonitis and secondary lung cancer. Although risk of radiation pneumonitis is already low, further reduction of risk may be achieved with normal tissue-sparing radiation techniques. Grantzau et al. [15] found that the risk of secondary lung cancer after breast irradiation mainly increases for smokers, and the median time from breast cancer treatment to secondary lung cancer diagnosis was 12 years. Although the absolute risk is relatively low, the growing number of long-term survivors after breast cancer treatment highlights the need for advances in normal tissue-sparing radiation techniques. In our study, DIBH resulted in reduction of mean heart dose and $V_{56 y}$ volumes compared with FB. Our results are concordant with previous studies: Conway et al. [10] found that DIBH could reduce heart exposure in right breast irradiation. In addition, Pedersen et al. [16] found that DIBH could eliminate cardiac volume within RT fields in nearly all patients. However, Essers et al. [9] reported that DIBH did not result in a relevant dose reduction to the heart. The heartsparing effect of DIBH in patients with LBC is well established and significant absolute dose reduction can be achieved with this technique. Although cardiac exposure in patients with $\mathrm{RBC}$ is already low, every effort should be made to obtain maximal cardiac protection, as even small reductions in cardiac exposure could have clinically relevant, long-term benefits.

DIBH does not require substantial cost to implement. A study from India showed that DIBH is cost-effective in LBC patients to reduce cardiac complications [17]. We are aware that this technique may not be as cost-effective in RBC as in LBC, but these are first steps and we believe that it is too early to comment definitively on cost effectiveness of the technique in RBC patients.

The DIBH technique has some limitations. Firstly, the technique is time-consuming, since setup is more complex and treatment delivery lasts longer. We hope that eventually the time needed for DIBH setup will be shortened with gained experience. However, if we are aiming to spare the surrounding organs and reduce morbidity and mortality related to RT, we may have to accept this limitation of the method. Chatterjee 
et al. [17] demonstrated that approximately 3.57 person-hours were necessary per Gy of reduction in mean heart dose, and the excess years of life lost from ischaemic heart disease if DIBH was not done was 0.95 per 100 patients, which translates into a saving of 12.8 hours of life saved per person-hour of work in implementing DIBH. Another important drawback of the technique is the need for patient cooperation.

In conclusion, in $\mathrm{RBC}$ patients $\mathrm{DIBH}$ is an effective technique to reduce liver, ipsilateral lung and heart exposure to radiation.

\section{Conflict of Interest}

No potential conflict of interest relevant to this article was reported.

\section{References}

1. EBCTCG (Early Breast Cancer Trialists' Collaborative Group). Effect of radiotherapy after mastectomy and axillary surgery on 10-year recurrence and 20-year breast cancer mortality: meta-analysis of individual patient data for 8135 women in 22 randomised trials. Lancet 2014;383:2127-35.

2. Darby SC, McGale P, Taylor CW, Peto R. Long-term mortality from heart disease and lung cancer after radiotherapy for early breast cancer: prospective cohort study of about 300,000 women in US SEER cancer registries. Lancet Oncol 2005;6:557-65.

3. Darby SC, Ewertz M, McGale $P$, et al. Risk of ischemic heart disease in women after radiotherapy for breast cancer. $\mathrm{N}$ Engl J Med 2013;368:987-98.

4. Sixel KE, Aznar MC, Ung YC. Deep inspiration breath hold to reduce irradiated heart volume in breast cancer patients. Int J Radiat Oncol Biol Phys 2001;49:199-204.

5. Stranzl H, Zurl B, Langsenlehner T, Kapp KS. Wide tangential fields including the internal mammary lymph nodes in patients with left-sided breast cancer: influence of respiratory-controlled radiotherapy (4D-CT) on cardiac exposure. Strahlenther Onkol 2009;185:155-60.

6. Vikstrom J, Hjelstuen MH, Mjaaland I, Dybvik KI. Cardiac and pulmonary dose reduction for tangentially irradiated breast cancer, utilizing deep inspiration breath-hold with audiovisual guidance, without compromising target coverage. Acta Oncol 2011;50:42-50.

7. Nissen HD, Appelt AL. Improved heart, lung and target dose with deep inspiration breath hold in a large clinical series of breast cancer patients. Radiother Oncol 2013;106:28-32.

8. Bruzzaniti V, Abate A, Pinnaro $P$, et al. Dosimetric and clinical advantages of deep inspiration breath-hold (DIBH) during radiotherapy of breast cancer. J Exp Clin Cancer Res 2013;32:88.

9 Essers M, Poortmans PM, Verschueren K, Hol S, Cobben DC. Should breathing adapted radiotherapy also be applied for right-sided breast irradiation? Acta Oncol 2016;55:460-5.

10. Conway JL, Conroy L, Harper $L$, et al. Deep inspiration breathhold produces a clinically meaningful reduction in ipsilateral lung dose during locoregional radiation therapy for some women with right-sided breast cancer. Pract Radiat Oncol 2017;7:147-53.

11. Offersen BV, Boersma $\sqcup$, Kirkove $C$, et al. ESTRO consensus guideline on target volume delineation for elective radiation therapy of early stage breast cancer. Radiother Oncol 2015;114:3-10.

12. RTOG Foundation Inc. Breast cancer contouring atlas [Internet]. Philadelphia, PA: RTOG Foundation Inc.; c2019 [cited 2019 Aug 15]. Available from: http://www.rtog.org/CoreLab/ ContouringAtlases/BreastCancerAtlas.aspx

13. Hagen $B$, O'Beirne $M$, Desai $S$, Stingl M, Pachnowski CA Hayward S. Innovations in the ethical review of health-related quality improvement and research: the Alberta Research Ethics Community Consensus Initiative (ARECCI). Healthc Policy 2007;2:e164-77.

14. Hormati A, Hajiani E, Alavinejad P, Shayesteh AA, Masjedizadeh $A R$, Hashemi SJ. Evaluation of breast cancer radiotherapy induced liver fibrosis by elastography. J Gastroenterol Hepatol Res 2014;3(8):1026-209.

15. Grantzau T, Thomsen MS, Vaeth M, Overgaard J. Risk of second primary lung cancer in women after radiotherapy for breast cancer. Radiother Oncol 2014;111:366-73.

16. Pedersen AN, Korreman S, Nystrom H, Specht L. Breathing adapted radiotherapy of breast cancer: reduction of cardiac and pulmonary doses using voluntary inspiration breath-hold. Radiother Oncol 2004;72:53-60.

17. Chatterjee $S$, Chakraborty S, Moses $A$, et al. Resource requirements and reduction in cardiac mortality from deep inspiration breath hold (DIBH) radiation therapy for left sided breast cancer patients: a prospective service development analysis. Pract Radiat Oncol 2018;8:382-7. 